\title{
MANX, A 6-D MUON COOLING DEMONSTRATION EXPERIMENT*
}

\author{
Thomas Roberts, Mohammad Alsharo'a, Pierrick M. Hanlet, Rolland Johnson, Moyses Kuchnir, \\ Kevin Paul, Muons, Inc., Batavia, Illinois, U.S.A. \\ Charles Ankenbrandt, Alfred Moretti, Milorad Popovic, Victor Yarba, \\ Fermilab, Batavia, Illinois, U.S.A. \\ Daniel Kaplan, Katsuya Yonehara, Illinois Institute of Technology, Chicago, Illinois, U.S.A.
}

\begin{abstract}
Most ionization cooling schemes now under consideration are based on using many large flasks of liquid hydrogen energy absorber. One important example is the proposed Muon Ionization Cooling Experiment (MICE), which has recently been approved to run at the Rutherford Appleton Laboratory (RAL). In the work reported here, a potential muon cooling demonstration experiment based on a continuous liquid energy absorber in a helical cooling channel (HCC) is discussed. The original HCC used a gaseous energy absorber for the engineering advantage of combining the energy absorption and RF energy regeneration in hydrogen-filled RF cavities. In the Muon And Neutrino eXperiment (MANX) that is proposed here, a liquid-filled HCC is used without RF energy regeneration to achieve the largest possible cooling rate in six dimensions. In this case, the magnetic fields of the HCC must diminish as the muons lose momentum as they pass through the liquid energy absorber. The length of the MANX device is determined by the maximum momentum of the muon test beam and the maximum practical field that can be sustained at the magnet coils. We have studied a 3 meter-long HCC example that could be inserted between the MICE spectrometers at RAL.
\end{abstract}

\section{INTRODUCTION}

In order for the high energy physics community to accept the idea of actually constructing a neutrino factory or a muon collider, it is necessary to demonstrate both the physics and the engineering feasibility of the special components required in their construction. As muons are inherently generated with a very large emittance, a key new component of such a facility is equipment to reduce their emittance to the acceptance of an affordable accelerator; this is known as beam cooling. Due to the short lifetime of the muon, the only suitable method for this is ionization cooling [1]. There are many variations on ionization cooling, and the key innovation discussed here is to combine a long continuous energy absorber with a helical magnetic channel to provide not only a rather large cooling factor, but also cooling in all six dimensions of the beam distribution.

\section{THE HELICAL COOLING CHANNEL}

The Helical Cooling Channel (HCC) [2] consists of three superimposed superconducting magnets that provide solenoid, helical dipole, and helical quadrupole fields, plus a continuous energy absorber along the helical magnetic channel. By tailoring the magnetic fields to the muons' energy loss in the absorber, the muon beam can be kept in the magnetic channel as it cools and loses energy. The key design challenge is to maintain the proper relationships among the different components of the field so the muons remain in the helical channel, the desired dispersion is maintained, and the acceptance is as large as possible. The dispersion is the correlation between momentum and transverse position, and in a helical channel it determines the relationship between the muon path length and momentum. That relationship is the essential design parameter that determines the emittance exchange and therefore the longitudinal cooling in the helical channel.

The dipole and quadrupole fields are shown in Figure 1 at the entrance plane of the HCC; in addition there is a larger solenoid field out of the paper. For successive planes into the paper (along the solenoid axis), the figure rotates clockwise around the center of the solenoid, so the acceptance follows the helix. The reference particle (centerline of the acceptance) is at the center of the blue circle, angled $45^{\circ}$ into the paper to the right, along the helix (the beam centerline is along the helix, not along the solenoid axis). The dipole field and the solenoid field (not shown) must be designed so that the reference particle follows the desired helix, which means they must decrease as muons lose energy by ionization loss in the absorber. The quadrupole field must vary accordingly to maintain the acceptance.

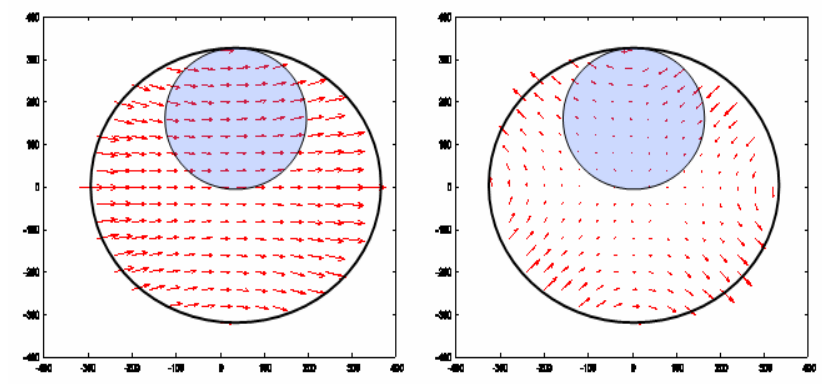

Figure 1. Helical Dipole (left) and Quadrupole (right) fields at the entrance to the HCC. The large circle is the solenoid (64 $\mathrm{cm}$ inner diameter), and the smaller blue circle is the region of acceptance of the HCC.

* Work supported by the U.S. DOE SBIR grant DE-FG02-04ER84015. 
The helical cooling channel described here is 3 meters long and $64 \mathrm{~cm}$ in diameter (plus the solenoid coils and their supports). The helix has a 1 meter period so the beam makes three turns around the helix inside the HCC. It is designed for a muon beam with a mean momentum of $300 \mathrm{MeV} / \mathrm{c}$, which loses energy in the liquid hydrogen absorber down to a mean momentum of $85 \mathrm{MeV} / \mathrm{c}$. At the entrance the solenoid field is 8.5 Tesla, the dipole field is 3.7 Tesla, and the quadrupole gradient is 7.7 Tesla/meter; all of these decrease along the HCC. These large field values make this a challenging magnet to construct, and a major part of our ongoing design effort will be to trade off the cooling performance with the practical cost and effort of constructing the channel.

The advantage of this HCC over other cooling demonstration approaches is shown in Figure 2: in a section only 3 meters long a reduction in the $6 \mathrm{~d}$ emittance by a factor of 2 can be achieved, with a third of the cooling being longitudinal. This is about 20 times more cooling than in the MICE experiment [3], which has no longitudinal cooling (an essential requirement for a muon collider).
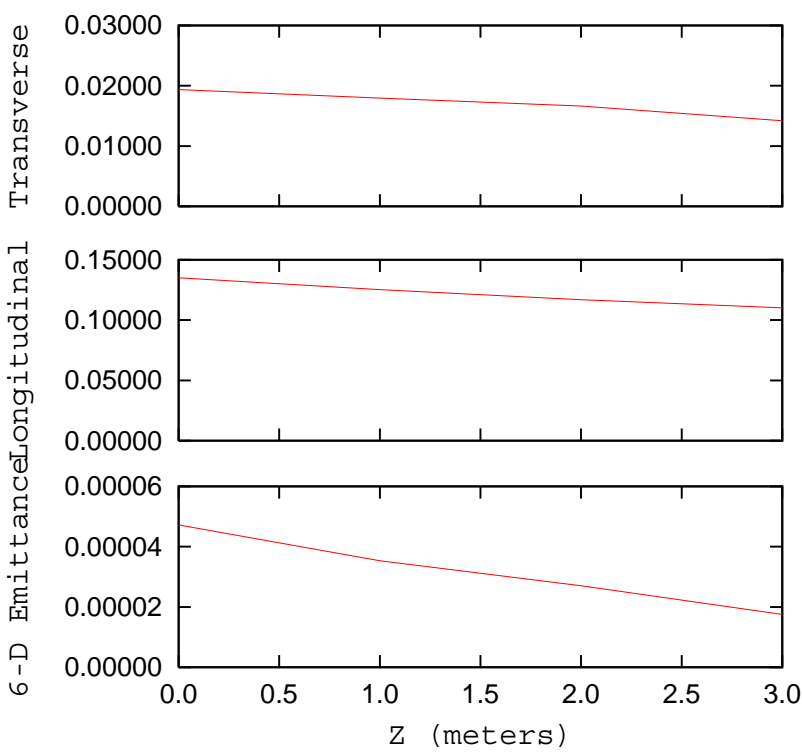

Figure 2. Emittance along the HCC, for transverse (top), longitudinal (middle), and $6 \mathrm{~d}$ emittance (bottom). The $\mathrm{Z}$ axis is position along the solenoid axis.

\section{EXPERIMENTAL CONCEPT}

The basic concept of the experiment is to put a muon beam into a spectrometer upstream of the cooling channel, then through the HCC, and then into another spectrometer downstream of the cooling channel. By measuring individual muon tracks both upstream and downstream of the HCC a "virtual bunch" can be constructed offline and its emittance before and after the HCC can be computed. This then gives a direct measure of the emittance reduction actually achieved in the channel.
While there are several possibilities for implementing the experiment, at present an attractive possibility is to reuse the beamline and spectrometers being constructed for the MICE experiment. This beamline should be able to provide at least a hundred muon events per second, and the spectrometers have an acceptance and a resolution more than adequate for our needs. A preliminary layout of a helical cooling channel with the MICE spectrometers is shown in Figure 3.

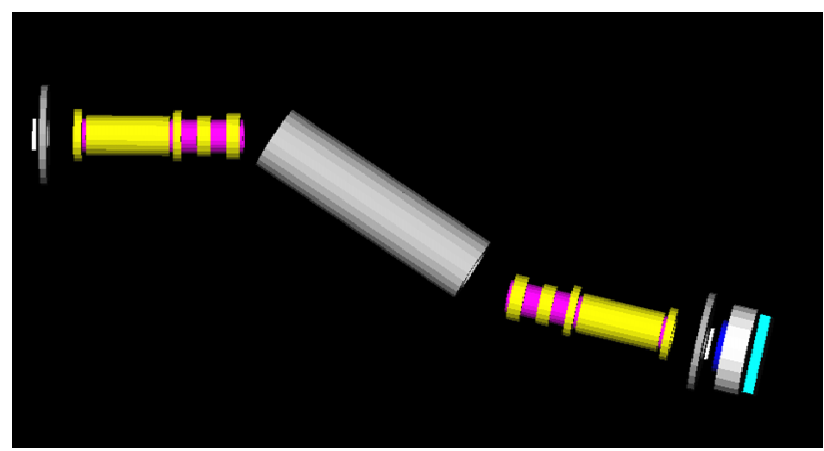

Figure 3. The MANX HCC (gray) with the MICE spectrometers and particle ID counters. The muon beam enters in the upper left. The spectrometer solenoid coils are yellow, surrounding a magenta beam pipe; at the lower right are a time-of-flight counter, a Cherenkov counter and an electron calorimeter (all for rejecting $\mu \rightarrow$ e decays).

The solenoid and HCC fringe fields are important in laying out the experiment. This is seen in Figure 4: the muon beam makes about $1 / 2$ of a turn around the "bent solenoid" fringe field between the upstream spectrometer and the HCC. A similar effect will occur downstream of the HCC, but the simulation of transport into the downstream spectrometer has not yet been completed. The muon beam must of course enter the $\mathrm{HCC}$ at the $45^{\circ}$ angle of its acceptance, but the fringe fields affect the beam so that the centerline of the HCC solenoid is only $32^{\circ}$ from the centerline of the first spectrometer. The location and orientation of the HCC was determined by tracking a reference particle down the centerline of the spectrometer through the fringe fields and into the centerline of the HCC acceptance. 


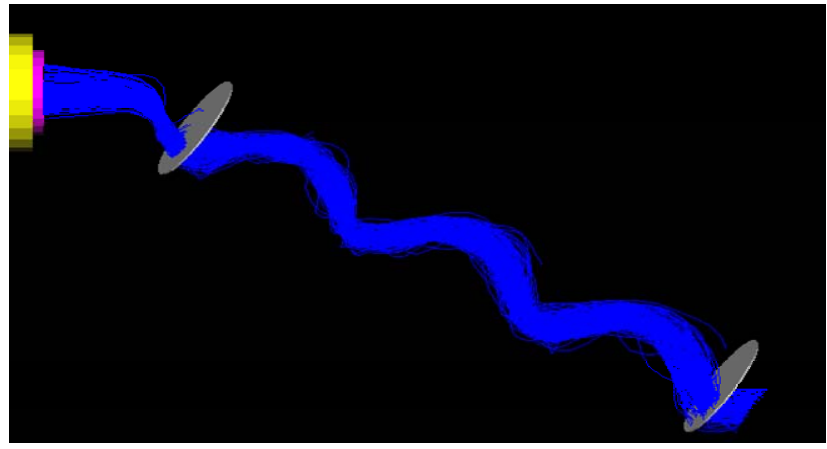

Figure 4. Muon tracks exiting the first spectrometer and traversing the Helical Cooling Channel (only its end caps are shown here for clarity). Transverse cooling occurs primarily in the angular distributions, not size of the beam, so it is not visible here.

\section{THE ABSORBER}

The energy absorber in an ionization cooling channel is a major factor in determining the cooling factor of the channel. Due to the tradeoff between energy loss (cooling) and multiple scattering (heating), there is a strong preference for the lowest- $Z$ material possible. The nominal design discussed here uses liquid hydrogen as the energy absorber. But that requires a large volume of hydrogen (over 250 liters of liquid $\mathrm{H}_{2}$ ), and safety concerns for a demonstration may make it more attractive to use liquid helium, in which case it could also be used to cool the superconducting coils of the HCC. Note that the muon energy loss is significant: this design has muons with momentum $300 \mathrm{MeV} / \mathrm{c}$ entering the $\mathrm{HCC}$ and $85 \mathrm{MeV} / \mathrm{c}$ exiting, which corresponds to a total energy loss of $57 \%$, and a kinetic energy loss of $86 \%$. In ionization cooling the overall cooling factor increases with the ratio of energy loss to incident energy, and this large fractional energy loss is what gives this channel its large cooling factor. As the muons follow helical trajectories through the absorber, their path length is $\sqrt{2}$ times the length of the HCC.

\section{BEAM MATCHING}

An important aspect of the design of this experiment is matching the beam into and out of the HCC. At present work is only beginning on this effort. It is important to demonstrate the ability to transport a muon beam through the HCC with minimal loss and with little or no emittance growth, as that will be important in any future facility. The MICE spectrometers have two matching coils at their inside ends (i.e. nearest the $\mathrm{HCC}$ ), which should provide enough flexibility to achieve a good match at each end of the HCC.

\section{EXPERIMENTAL MEASUREMENTS}

The multifunction magnet of the HCC will be built with separate windings for the solenoid, helical dipole, and helical quadrupole fields. This will provide flexibility in tuning the channel to vary the mixing between transverse and longitudinal cooling, and a wide range of ratios will be possible. In addition, we will probably segment the coils along $\mathrm{z}$ so we can vary the required energy loss profile of the absorber and thus accommodate different absorber materials. This will give us the ability to configure the channel in many different ways. Using the MICE beamline and spectrometers, we should be able to achieve at least 100 good muons per second, so high statistics should be possible in a few hours of data taking for each configuration. The results will almost surely be limited by systematic errors, so a considerable fraction of the beam time will be devoted to exploring and measuring them.

\section{CONCLUSION}

A neutrino factory, and especially a muon collider, would be a powerful new facility for answering some of the major questions of particle physics today [4]. To make either one a reality requires a realistic demonstration of both the physics and engineering of an ionization cooling channel, including actual operation in a muon beam. This experiment, with its helical cooling channel, will be able to do that in ways complementary to other demonstration experiments: besides providing another demonstration of transverse cooling with a different engineering solution, MANX will verify emittance exchange and 6-d cooling in a HCC while serving as a prototype of a precooling device for a high-intensity muon beam line.

\section{REFERENCES}

[1] D. Neuffer, AIP Conference Proceedings -- January 20, 1996 -- Volume 352, Issue 1, pp. 12-18.

M. M. Alsharo'a et al., Phys. Rev. ST Accel. Beams 6, 081001 (2003)

[2] Y. Derbenev and R. P. Johnson, Phys. Rev. ST Accel. Beams 8, 041002 (2005)

[3] The MICE experiment, http://hep04.phys.iit.edu/mice/

Note that several of the authors of this paper are active members of the MICE collaboration.

[4] The most recent thoughts from a variety of authors are available from the plenary sessions of NuFact04, http://www-kuno.phys.sci.osaka-u.ac.jp/ nufact04/ agenda.html

C. Albright et al, http://arxiv.org/abs/hep-ex/0008064

C. Quigg, http://arxiv.org/abs/hep-ph/9803326 